\title{
Introgression of Striga Resistance Genes into a Sudanese Sorghum Cultivar, Tabat, Using Marker Assisted Selection (MAS)
}

\section{Yasir Ahmed Gamar* and Abdalla Hassan Mohamed}

\author{
Sorghum Breeding and Genetics Research Program, Agricultural Research Corporation, ARC, \\ Wad-Medani, Sudan, P. O. Box 126.
}

\section{ARTICLE INFO}

Article No.: 061013654

DOI: 10.15580/GJAS.2013.7.061013654

Submitted: 10/06/2013

Accepted: 22/07/2013

Published: 29/07/2013

*Corresponding Author

Yasir Ahmed Gamar

E-mail: gamarotta@yahoo.com

Phone: +2499-128-47869

Keywords:

Quantitative Trait Loci (QTLs), Striga, Sorghum, Marker Assisted Selection (MAS), Sudan

\section{ABSTRACT}

Resistant crop cultivars provide the most economical, practical and sustainable method of Striga control. However, the development of resistant sorghum cultivars slowed by the complexity of the environment and the host/parasite interactions, which made field resistance erratic and unreliable. Recent adventure of molecular markers to tag gene(s) that confer important traits offers new hope for Striga control. Significant progress has been made to identify molecular markers linked to Striga resistance in sorghum variety, N13. Five genomic regions (QTLs) associated with Striga resistance were identified. Flanking simple sequence repeats (SSRs) markers to each QTL were also identified and made available.

The aim of this study was to transfer Striga resistance from, N13 to an elite farmer preferred sorghum cultivar, Tabat. Generations of F1, BC1F1, BC1S1 and BC2F1 populations were developed. F1 and BC1F1 generations were first genotyped and progenies with two or more QTLs were backcrossed to generate BC1F1 and $\mathrm{BC2F1}$, respectively. BC2F1 were further genotyped and progenies with two different QTLs were intercrossed for foreground selection. BC2F1 progenies were selfed to generate BC2S1, BC2S2 population. 19 progenies, BC1S1, with Striga resistance QTLs were tested in Striga artificial infested plots. Progenies with the two or more QTLs showed high levels of Striga field resistance, confirming the effectiveness of marker assisted selection (MAS). Significant differences were observed among the progeny tested in the level of Striga resistance and other agronomical traits. 


\section{INTRODUCTION}

Sorghum [Sorghum bicolor (L) Moench, $2 n=20$ ] is one of the most important cereal crops and well adapted to harsh environments characterized by drought and high temperatures. It was first domesticated in the region of North East Africa (Doggett, 1988).

The witch-weed (Striga spp.), an obligate root parasite of various crops, is the major constrain to sorghum production in rain-fed areas of semi-arid tropics (Ejeta, 1992). The parasite result a considerable crop damages, especially in the semi arid-tropic of the world (Rich, et al., 2004). Striga causes severe reduction to the host crops. Losses in crop yield due to Striga infestation are estimated in millions of tones annually (Olivier, 1995). In Sorghum yield, losses may reach $100 \%$ on heavily infested soils (Parker and Riches, 1993).

The parasite control is difficult, but it is not impossible. In the last few decades, efforts have been devoted to develop methods for Striga control. The most promising way for controlling the parasite resides on the development of resistant cultivars (Ejeta, 1991). However, breeding efforts were hampered by the lack of knowledge for the genetics of resistance and lack of reliable and rapid screening methods (Ejeta, 1992). Screening for Striga resistance in the field has failed and slowed due to the quantitative nature of the trait and the influence of the environment. Screening genotypes in Striga infested fields is often inefficient due to the host complexity, parasite and environment interactions (Ejeta 1992). Integrated Striga management approach was also suggested by Ramiah (1987). He suggested the use of resistant lines, in combination with cultural practices and chemical inputs. The integrated management will not only provide effective Striga control but also suppresses development of virulent strain of the parasite and reduces the build up of seeds in the soil seed bank (Ejeta, et al., 1991).

Molecular Markers or genetic markers are DNA sequences associated with certain parts of the genome and they have well defined phenotypes. They can also be defined as, DNA pieces that flag the presence or absence of particular traits (Paterson, 1996). Considering their abundance, molecular markers are powerful tools that speed and increase the precision and the effectiveness of plant breeding (Paterson, 1996). Molecular markers allow selection for traits on basis of a simple laboratory tests on a small plant tissue, rather than direct measurements of the character itself. Molecular makers also showed great potential in selection and manipulation of genotypes for desirable quantitative traits loci (QTLs) that condition complex economic traits (Ejeta, et al., 2000).

Marker assisted selection (MAS) is defined as the process of using the results of DNA testing to assist in the selection of desirable individuals, for specific trait, to become the parents for the next generation (Eenennaam, 2004). Moreover, the development of molecular markers for genetic analysis expanded the circle of knowledge of genetics and understanding of the structure and behavior of organism genomes (Korzun, 2005). To date, most of modern crop varieties are developed through marker assisted breeding and genetic engineering (Ejeta, 1992). In the last few decades, rapid progress has been made in molecular techniques and great amount of polymorphic markers are generated, sufficient enough to build dense linkage maps (Korzun, 2005). Significant progress has been made in identifying markers and quantitative trait loci (QTLs) associated with Striga resistance. Three major QTLs that associate with Striga resistance were identified in sorghum cultivar, N13.

The objective of this study was to transfer Striga resistance from $\mathrm{N} 13$ into farmer preferred sorghum cultivar 'Tabat' using the strength of marker assisted selection and to verify the effectiveness of the marker assisted selection as a breeding tool and generation of elite lines for future use.

\section{MATERIAL AND METHODS}

\section{Germplasm}

The Striga susceptible sorghum cultivar 'Tabat' was selected as recipient parent and Striga resistance sorghum variety, $\mathrm{N}-13$, as donor parent. Backcrossing method of breeding was used. Achieving effective backcrossing and optimum population size; $8 \mathrm{~F} 1 \mathrm{~s}$ (Four of them were reciprocal cross), $168 \mathrm{BC} 1 \mathrm{~F} 1,168$ $\mathrm{BC} 2 \mathrm{~F} 1, \mathrm{BC} 2 \mathrm{~S} 1$ progenies were generated (Fig.2). Confirming the effectiveness of marker assisted breeding 19 Tabat BC1S1 lines carrying different QTLs were field tested for foreground and background selection.

\section{Mapping Of Striga Resistance Genes}

This study was based on a published sorghum genetic linkage map constructed by Haussmann, et al. (2001b) (Fig.1).

\section{Genotyping}

The genotyping work was carried out at Biosciences for Eastern Central Africa (BecA) molecular biology laboratory located at the International Livestock's Research Institute (ILRI), Nairobi. DNA extraction method was used, as described by Mace et al., (2004).

Genotyping process which can be defined as laboratory method used to detect and amplify a known polymorphic DNA nucleotides sequence, marker, in the generated population individuals. The parents; N13, and Tabat and his $\mathrm{F} 1 \mathrm{~s}, \mathrm{BC} 1$ and $\mathrm{BC} 2$, derived progenies were screened with 11 SSRs markers txp201, txp15, txp225, txp65, txp302, txp303, txp145, Xtxp57, txp208, txp50 and txp304, that have close association with Striga resistance regions QTLs. SSRs amplification was performed according to the method 
described by Haussmann, et al., 2001. Then the amplified samples (PCR products) were prepared with

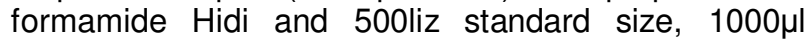
to $12 \mu \mathrm{l}$ respectively, of which $0.7 \mu \mathrm{l}$ of the amplified DNA transferred to the sequencer. Scoring of peaks achieved using gene-mapper v.4.0 software program.

\section{Bc1s1 Field Testing}

Selected BC1S1 (19 lines) derived progenies were screened under Striga artificially infested plot at ARC, Gezira Research Station. The plot artificially infested as follows: viable Striga seeds mixed with soil $(1 \mathrm{~g}$ of Striga seeds/ $1 \mathrm{~kg}$ soil). The mixture evenly distributed in holes on top of $80 \mathrm{~cm}$ ridges ( $1 \mathrm{~g}$ soil seed mixture/ $\mathrm{m}^{2}$ ). Few days following the artificial infestation, sorghum was planted inter-row spacing $1 / 10 \mathrm{~cm}$. Number of emerged Striga plant was evaluated 45 days after crop emergence. The progenies evaluated for days to $50 \%$ flowering, plant height and weight of 100 seed. Randomized complete block design (RCBD) with three replicates was used.

\section{RESULTS}

\section{Introgression of Resistance Genes}

The presence of the regions, QTLs, in association with Striga resistance is detected through the presence or absence of the associated polymorphic flanking markers. Eight F1s (Tabat $\times \mathrm{N}$-13) progenies were generated then $168 \mathrm{BC} 1 \mathrm{~F} 1$ progenies were generated and screened with 10 SSRs polymorphic markers. The screened progenies, 145, showed the absence of regions, QTLs, associated Striga resistance. Which distributed as, 52 showed no reaction with primers, 93 progenies showed the reaction but the absence of polymorphisms in the flanking markers. 23 progenies showed the presence of one or more of the three major regions, QTLs, linked to Striga resistance. Indicating the presence of at least one region associated with Striga resistance in these progenies. 18 of the progenies showed the presence of the QTLs, flanking markers in heterozygous status. Only 5 progenies showed the presence of the QTLs, polymorphic flanking markers, as homozygous status.

Progenies of $\mathrm{BC} 2 \mathrm{~F} 1$ with the target regions, QTLs, were advanced to generate BC1F1. 168 derived progenies, BC2F1, were generated. Progenies were screened with 7 SSRs polymorphic markers to detect the presence of Striga resistance regions, QTLs. 115, showed the presence of the major three QTLs, in close association with Striga resistance, which distributed as; 36 progenies showed the presence of QTLs, polymorphic flanking markers, as heterozygote and 79 progenies showed the presence of, QTLs, polymorphic flanking markers, as homozygous status, indicating the presence of at least one region associated with Striga resistance in these progenies. 53 progenies showed the absence of these regions, QTLs.

\section{Foreground Selection}

The recurrent susceptible parent, Tabat and 19 BC1S1 derived lines (Table 1) selected from the genotyping results carrying different type of resistance (different letters), four parameters were majored; 1) Striga count 2) plant height 3) $50 \%$ Flowering and; 4) 100 seed weight.

Field testing results showed significant differences in maturity among $\mathrm{BC} 1 \mathrm{~S} 1$ progenies and the susceptible parent Tabat. However, no significant differences were observed between progenies BC1S1144-D and BC1S1-127-R (Table 1). The least significant differences (LSD) at $\mathrm{P}=0.05$ was used to separate means.

Striga Count: No significant differences were observed between the susceptible progeny $\mathrm{BC} 1 \mathrm{~S} 1$ 127-R and Tabat (two controls), and BC1S1-121- $\mathrm{H}$, BC1S1-62-D, BC1S1-96-H, BC1S1-75-D and BC1S194-H. Significant differences were observed for Striga count among these progenies, BC1S1-107- $\mathrm{H}, \mathrm{BC} 1 \mathrm{~S} 1-$ 67-H, BC1S1-110-H, BC1S1-16-H, BC1S1-24-H, BC1S1-144-D, BC1S1-138-D, BC1S1-55-H, BC1S152-H, BC1S1-19-D, BC1S1-63-H, BC1S1-122-H and BC1S1-2-D, (Table 1). The least significant differences (LSD) at $P=0.05$ was used to separate means.

Plant Height $(\mathrm{cm})$ : The results showed significant differences in all selected progenies (treatments), comparing with the susceptible checks, Tabat and BC1S1-127-R (Table 2). The least significant differences (LSD) at $\mathrm{P}=0.05$ was used to separate means.

Weight of 100-Seed (g): results of the hundred-seed weight showed no differences among the BC1S1-62-D, BC1S1-196-H, BC1S1-144-D, BC1S1-19-D, BC1S1-63-H and BC1S1-122-H progenies. However, progenies, $\mathrm{BC} 1 \mathrm{~S} 1-121-\mathrm{H}$, BC1S1-107-H, BC1S1-67-H, BC1S1-110-H, BC1S116- H, BC1S1-75-D, BC1S1-24-H, BC1S1-138-D, BC1S1-55-H, BC1S1-52-H, BC1S1-94-H and BC1S12-D, showed significant differences comparing with the susceptible checks (Table 1). The least significant differences (LSD) at $P=0.05$ was used to separate means.

\section{DISCUSSIONS}

Breeding for resistance in sorghum is recognized as the most feasible method for Striga control (Ejeta, 1992). However, developing crop cultivars with durable Striga resistance is slowed for the lack of a reliable screening method. Field screening is affected by many confounding environmental factors. In this era of biological sciences efforts were made to track important gene(s) that govern important traits. Genetic linkage maps were constructed; molecular markers and QTLs were placed on these maps for most crops. In sorghum, great efforts were made to construct consensus linkage maps, as well as to identify molecular markers and QTLs with close association to Striga resistance genes. These markers and QTLs will not only ease the screening for Striga resistance, but also help tracking important Striga resistance genes.

Indeed, in this study 11 markers were used for foreground selection. SSRs alleles were scored for 
absence and presence to detect Striga resistance QTLs. Each QTL region should be flanked at least by two markers to confirm its presence. Consistent with the literature that each chromosome should be checked with two to three makers and that would be efficient to control the confidence intervals and minimize the risk of losing the target alleles QTLs (Hospital and Charcosset, 1997).

The backcrossing method is generally used in plant breeding programs to transfer target allele(s) from a donor genotype, which mostly possesses undesirable and poor agronomic traits, into preferred genotypes (Allard, 1960). The method drawbacks, some of the donor parent inferior trait alleles transfer with the target alleles. The invention of marker assisted selection increased the precision of the backcrossing method to transfer certain alleles and reduce the probability of transferring the untargeted trait alleles.

To reduce the cost, F1 generation was not genotyped; the seed color of the hybrid was used as a physiological marker to detect $\mathrm{F} 1$ plant generation (spreader gene). $168 \mathrm{BC} 1 \mathrm{~F} 1$ and $\mathrm{BC} 2 \mathrm{~F} 1$ progenies were developed for detecting the presence of the major Striga resistance QTLs in these derived progenies. This population size corresponding with Hospital and Charcosset (1997) suggested population size. They stated that, introgression of three QTLs carrying chromosomal segments has proved to be possible by marker assisted selection with reasonable population size. Backcrossing scheme advanced to $\mathrm{BC}$, which recover only $87.5 \%$ of recurrent parent genome. This is not recovering most of recurrent parent genome but also reduce the chance of losing the maker in the recombination process in each generation, considering the large confidence interval in our mapping population. This is consistent with marker assisted background selection that achieved on $\mathrm{BC} 2$ and BC3 progenies by Bouchez et al. (2002).

Introgressing Striga resistance genes (QTLs) into Tabat BC1F1 derived progenies showed 20 progenies with the resistance one or more QTLs, which vary from 18 hybrids (heterozygous) five all donor parent QTLs and no QTLs were in 94 progenies. The few number of plant carrying all the target alleles might be due to crossing over, or might be associated with the based genetic map in which the flanking markers confidence interval vary from 25 to $40 \mathrm{~cm}$, this distance is long enough to allow passing the markers without the QTLs. Other studies stated that this reduction in expected frequencies over generations increased with the number of QTLs and the confidence interval between flanking markers. Tabat BC2F1 derived progenies results showed, 115 plants carrying Striga resistance QTLs, 79 progenies heterozygous QTLs, 36 donor resistant status QTLs. The QTLs are distributed over the A, B, I and J chromosome and 52 plants with no Striga resistance. This number is higher than the expected frequencies, it might also due to the above mentioned factors.

Reports suggested foreground selection is efficient for markers associated with three target regions without the help of phenotypic assays
(Bouchez, et al., 2002). At each backcross generation, the selection of the individuals that carry the allele of interest at the specific QTL is based on their genotype at neighboring markers(s) and then among these individuals one or several individuals carrying the most alleles of recipient type at markers are selected in the next step (Hospital and Charcosset, 1997). The information from genetic map can be used to identify, manipulate and complement traits to the maximum advantage (Paterson, 1996).

To confirm the effectiveness of marker assisted selection in introgressing of Striga resistance genes, 19 BC1S1 derived progenies were tested, under Striga artificial infestation, with the donor parent, Tabat, as a susceptible control. The susceptible control, Tabat, showed no flowering even after one hundred and twenty days from germination; it was wiped out by end of the season. The results confirmed the high susceptibility of the cultivar, Tabat, and high level of infestation in the artificially infested plot. BC1S1-127-R derived progeny, with no Striga resistance QTLs, showed levels of infestation comparable to that of Tabat. Consistent with early reports, sorghum yield losses may reach $100 \%$ on heavily infested soils (Parker and Riches, 1993). The results also indicated significant differences in 50\% flowering in the other 17 progenies carrying different Striga resistance QTLs in homozygous and heterozygous form ( $\mathrm{D}$ and $\mathrm{H}$ form of resistance). BC1S1-144-D derived progeny showed earliest 50\% flowering value. It might be generally stated that high levels of Striga infestation, delay and/or inhibit genotypes flowering. Susceptible genotypes early flower, intermediate genotypes with delayed flowering and resistant genotypes normal flowering. Emerged Striga count is considered as one of the field measurements that determine host plant resistance. Our data indicated that the susceptible checks, Tabat and BC1S1-127-R, have the lowest number of the emerged Striga plants in the genotypes tested. This is mainly due to poor development of the two checks as result of Striga damage, where they were completely wiped out at the end of season. Genotypes that carry different genetic resistance (QTLs), showed different numbers of emerged Striga plants. Significant differences were observed in plant height among these genotypes; the susceptible ones are poorly developed and stunted, although genotypes with resistance have normal development and plant height. These results correspond with many previous studies concerning Striga-host interactions. The study also measuring the hundred seed weight (which considered as one of the yield component), the results (Table1) showed no seeds for the susceptible parent Tabat (highly affected with Striga damage), 6 of the tested lines showed no significant differences, nevertheless, 12 lines carrying different genetic types of resistance showed a significant difference.

Appearance of the lines carrying group 5 (chromosome J-2QLTs-) as considerable amount and showing field resistance, this can give more attention for this region. In contrast with the study in Kenyan 
which minor genes showed more important with the Kenyan Striga seeds (Haussmann et al, 2001a).

The study confirmed the effectiveness of marker assisted selection (MAS) as screening for Striga resistance tool in sorghum. Tabat-derived progeny BC1S1-127-R (classified as susceptible by the genotyping data) showed the same level of susceptibility as the recurrent parent, Tabat. Tabatderived progenies BC1S1-67-H, BC1S1-110-H and $\mathrm{BC1S1-16-H}$ (classified as resistance by the genotyping data) showed not only high Striga resistance levels but also superior agronomic performance.

The data indicated that the Heterozygous $(\mathrm{H})$ status, showed effective transferring and expressing of Striga resistance genes as the homozygous (D), which confirm the dominant nature of Striga resistance in the donor parent, N13.

Field results showed stronger Striga resistance reactions in group 5 (2QTLs) than the other
QTLs groups. This indicates that marker assisted selection might also be reliably used not only to transfer QTLs regions but also might be used to weigh the contribution of each QTL to the trait of interest, even in a fairly saturated linkage map.

Striga field resistance is affected by many environmental factors as indicated by many reports. Our data also showed emerging Striga plant count to determine the level of Striga infestation in the field might be misleading unless it combined with other agronomic traits and yield components. This also showed the urgency to use MAS which is environment insensitive and for more precise screening to boost breeding for Striga resistance.

Pyramiding of Striga resistance genes into elite backgrounds is becoming possible through foreground and background selection in MAS breeding.

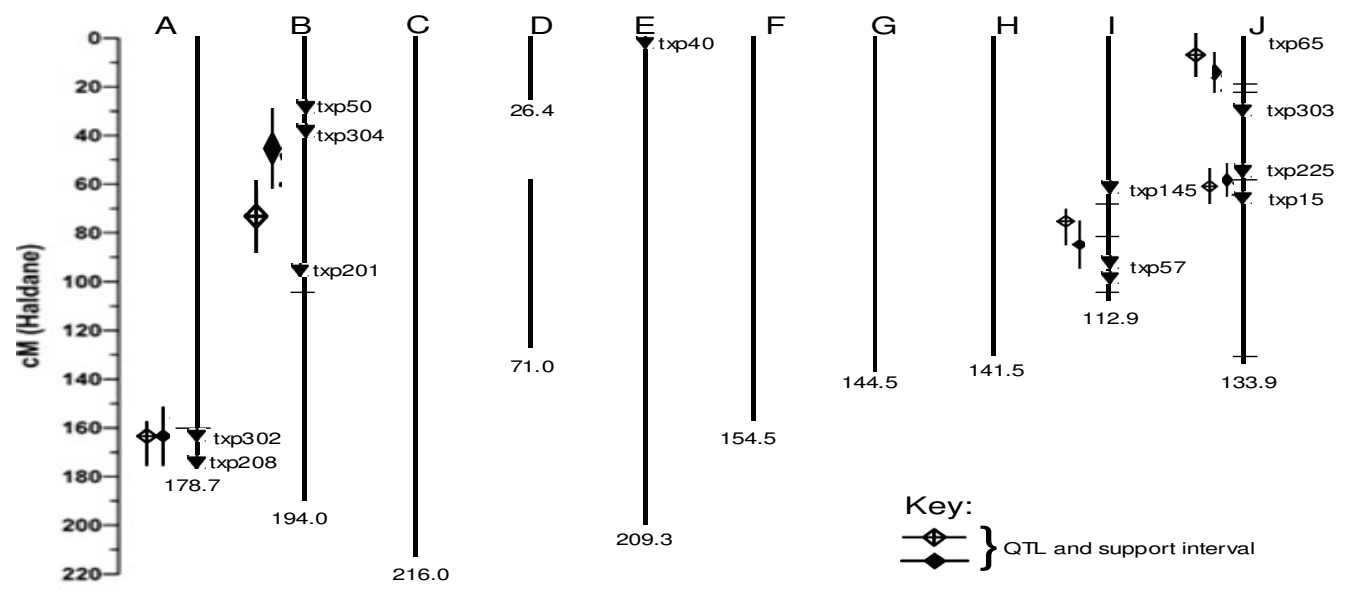

Figure (1) Major QTLs and SSR markers associated with Striga resistance in sorghum

FPSV Tabat X N13

F1 (Tabat X'N13) X Tabat

BC1F1 (genotyped) X Tabat



Figure (2) Crossing scheme: 
Table (1) Tabat BC1S1 field result

\begin{tabular}{|l|l|l|l|l|}
\hline Entries/treat & $\begin{array}{l}\text { Days to 50\% } \\
\text { Flowering }\end{array}$ & $\begin{array}{l}\text { Striga Count } \\
\text { (plant/20m }{ }^{2}\end{array}$ & $\begin{array}{l}\text { Plant Height } \\
(\mathrm{cm})\end{array}$ & $\begin{array}{l}\text { Weight of 100- } \\
\text { Seed }(\mathrm{g})\end{array}$ \\
\hline Tabat & 0.0 & 58 & 52.1 & 0.00 \\
\hline BC1S1-127-R & 0.00 & 4 & $49.3^{*}$ & 0.00 \\
\hline BC1S1-121-H & $79^{*}$ & 84 & $109.0^{*}$ & $2.30^{*}$ \\
\hline BC1S1-62-D & $77^{*}$ & 77 & $111.6^{*}$ & 1.07 \\
\hline BC1S1-107-H & $74^{*}$ & $164^{*}$ & $70.4^{*}$ & $2.27^{*}$ \\
\hline BC1S-96-H & $56^{*}$ & 85 & $74.8^{*}$ & 0.00 \\
\hline BC1S1-67-H & $62^{*}$ & $214^{*}$ & $147.0^{*}$ & $3.60^{*}$ \\
\hline BC1S1-110-H & $63^{*}$ & $250^{*}$ & $142.1^{*}$ & $3.00^{*}$ \\
\hline BC1S1-16-H & $69^{*}$ & $132^{*}$ & $139.9^{*}$ & $2.87^{*}$ \\
\hline BC1S1-75-D & $71^{*}$ & 72 & $112.8^{*}$ & $2.60^{*}$ \\
\hline BC1S1-24-H & $46^{*}$ & $164^{*}$ & $103.9^{*}$ & $2.37^{*}$ \\
\hline BC1S1-144-D & 0.0 & $94^{*}$ & $66.8^{*}$ & 0.00 \\
\hline BC1S1-138-D & $78^{*}$ & $180^{*}$ & $81.5^{*}$ & $2.37^{*}$ \\
\hline BC1S1-55-H & $74^{*}$ & $484^{*}$ & $127.7^{*}$ & $3.37^{*}$ \\
\hline BC1S1-52-H & $77^{*}$ & $148^{*}$ & $111.4^{*}$ & $3.07^{*}$ \\
\hline BC1S1-19-D & $79^{*}$ & $107^{*}$ & $92.2^{*}$ & 1.33 \\
\hline BC1S1-63-H & $86^{*}$ & $202^{*}$ & $100.0^{*}$ & 0.77 \\
\hline BC1S1-94-H & $75^{*}$ & 56 & $100.8^{*}$ & $2.60^{*}$ \\
\hline BC1S1-122-H & $54^{*}$ & $100^{*}$ & $99.6^{*}$ & 1.30 \\
\hline BC1S1-2-D & $83^{*}$ & $206^{*}$ & $98.7^{*}$ & $2.30^{*}$ \\
\hline LSD & 14.36 & 81.40 & 20.41 & 0.56 \\
\hline C.V\% & 25.0 & 88.5 & 32.0 & 45.6 \\
\hline Overall Mean & 62.6 & 143.7 & 99.6 & 1.9 \\
\hline
\end{tabular}

${ }^{*}$ Significant at 0.05 probability level

Key:

$\mathrm{D}=$ Presence of the donor parent Striga resistance region in homozygous status, $\mathrm{H}=$ Presence of the donor parent Striga resistance regions in heterozygous status, R= Absence of the donor parent Striga resistance region

\section{ACKNOWLEDGEMENTS}

Authors are grateful to Prof. A. A. Hamada, Director General of Agricultural Research Corporation (ARC) of Sudan, BMZ Striga project, Bioscience Central and Eastern Africa (BecA) and University of Honhenhiem, for their morale, technical and financial support.

\section{REFERENCES}

Allard, R. (1960). Principles of plant breeding. Wiley, New York. Berner, D. K., J. G. Kling, and B. B. Singh. 1995. striga research and control. Plant Dis. 79:652-660.

Bouchez, A., F. Hospital, M. Causse, A. Gallais, and A. Charcosset. (2002). Marker-Assisted Introgresstion of Favorable Alleles at Quantitative Trait Loci Between Maize Elite Lines. Genet. p. 162:1945-1959.

Doggett, H. (1988). The Sorghum crop. In Sorghum (2nd ed). Longman group U.K. Limited, London, U.K. pp 368-404.

Eenenaam, A. V. (2004). Marker assisted selection backgrounder.

IN:

http://repositories.collib.org/anrrec/sfrec/2004_mar ker_assisted_selection_backgrounder.
Ejeta, G., P. Goldsbrough, M. Tuinstra, E. Grote, A. Menkir, Y. Ibrahim, N. Weerasuriya. A. MelakeBerhan, and C. A. Shaner. (2000). In: B. Haussmann, H. Geiger, D. Hess, C. Hash, and P. Bramel-Cox (Eds.). Application of molecular markers in plant breeding. Training manual for a seminar held at IITA, Ibadan, Nigeria, from 16-17 August 1999. International Crops Research Institute for the semi-Arid Tropics (ICRISAT), Patancheru 502324, Andhra Pradesh, India.

Ejeta, G., L.Butler, and A. Babiker. (1992). New approaches to the control of striga. In: G. Ejeta, L. Butler and Babiker, [eds.] striga research at Purdue Bulletin, pp 11-13.

Ejeta, G., L. Butler, D.Hess, and R. Vogler. (1991). Genetic and breeding strategies for striga resistance in sorghum. In: J. Ransom, L J. Musselman, A. Worshamand C. Parker, [eds] proceedings of the 5th International Symposium on Parasitic Weeds, CIMMYT Nairobi. P. 539.

Haussmann a, B., D. Hess, G. Omanya, B. Reddy, N. Seatharama, S. Mukuru, M. Kayentao, H. Welz, and H. Geiger. (2001). Towards Marker-Assisted Selection for Striga Resistance in Sorghum. 7th International Parasitic Weed Symposium. Nantes, France June 2001. p. 212.

Haussmann b, B. I., D. E. Hess, G. O. Omanya, B. V. Reddy, H. G. Welz, and H. H. Geiger. (2001). 
Major and Minor Genes for Stimulation of Striga hermonthica Seed Germination in Sorghum, and Interaction with Different Striga Populations. Crop Sci. p. 41:1507-1512.

Hommond-Kosack, K. E., and J. D. G, Jones. (1997). Resistance genes dependent plant defense responses. Plant Cell, 8:1773-1791.

Hospital, F., and A. Charcosset. (1997). Markerassisted introgression of quantitative trait loci. Genetics147:1469-1485.

Korzun, V. (2005). Molecular Markers and Their Application In Cereals Breeding. In: WWW.fao.org/BIOTECH/docs/korzun.pdf.

Mace ES, Hutokshi k, Buhariwalla, Crouch JH (2004) A high-throughput DNA Extraction Protocol for Tropical Molecular Breeding Programs
Olivier, A. (1995). Witchweed, a parasite of African cereal crops-biology and control methods. Agronomie. 15:517-525.

Parker, C. and C. Riches. (1993). Parasitic Weeds of the World: Biology and control. Wallingford: CAB International.

Paterson, A. H. (1996). An historical Perseptive. P. 15. In:Paterson, A. H. (ed) Genome Mapping in Plants. Academic Press Austin Texsas, USA.

Ramiah, K. V. (1987). Breeding cereal grains to withweed. In Parasitic Weeds in Agriculture (L.J. Musslemann(eds.) CRC Press Boca Raton, FL USA. Vol., 1:227-242.

Rich, P., C. Grenier, and G. Ejeta. (2004). Striga Resistance in the Wild Relative of Sorghum. Crop Science 44:2221-2229. 